\title{
Static Equilibrium under Uncertainty and Incomplete Markets
}

\author{
by Karl Borch *
}

\section{Introduction}

1.1. The first model of general economic equilibrium was presented by Walras [15] more than hundred years ago. As the model became generally known, it must have been clear that it was desirable to generalize it, and take uncertainty into account. The most visible markets at the end of the last century were probably the stock exchanges, where uncertainty obviously played an important part. Insurance was also well developed, and it was natural to assume that premiums were determined by market forces. It is possible that some economists attacked the problem, but apparently they were not successful. The literature from that period has little to say about economic risk, beyond general statements that there should be a price, so that supply of, and demand for risk-bearing services are equal.

One who may have missed the boat is Böhm-Bawerk. In his first book [6] he discusses the value one should assign to conditional claims. He observed for instance that if some property is stolen, the owner has the right to get it back if the thief is caught. The question is which value should the owner assign to this right. Apparently it did not occur to Böhm-Bawerk that this right also might have a market value. Had he asked the question, it would have led straight to the market premium for burglary insurance, which had been introduced in England about twenty years earlier.

1.2. In retrospect it seems that it should have been quite possible to construct an equilibrium model explaining how insurance premiums are determined by market forces, at the turn of the century. The key to the problem is the Bernoulli Principle, today usually refered to as " the expected utility hypothesis ", which was well known, and often discussed among mathematicians at the time. The paper Bernoulli [5] presented in St. Petersburgh was translated into German in 1896, and Czuber [8] in a much used textbook on probability, stated that Bernoulli Principle had become the "foundation of the modern theory of value". (Grundlage der Modernen Wertlehre). Most economists at the time seem however to have been unaware of this.

Marshall [10] seems to have been well aware of the Principle's possibilities. In his "Mathematical Appendix", he mentions Bernoulli's assumption, which we today would

* Professor of insurance economics, Norwegian School of Economics and Business Administration, Bergen. 
write $u(x)=\log x$, and refers to the other "interesting guess" by Bernoulli's contemporary Cramer, that $u(x)=\sqrt{x}$. Apparently it did not occur to Marshall that either guess, or any other concave function would open the way to get rid of the "evils of uncertainty", which Marshall discusses in connection with insurance.

1.3. It is surprising that no economists seem to have taken these hints and put the Bernoulli Principle to use. It was obviously possible to apply the principle before von Neumann and Morgenstern [11] had proved that it could be derived as a theorem by a simple axiomatic method. Actually this result had been proved more than 15 years earlier by Ramsey [12], but it did not seem to have made any stronger impact in Cambridge than Marshall's hints 40 years earlier. If one seeks an explanation of the late development of an economic theory of uncertainty, it may be that only the generation of economists who moved to the forefront of research after World War Two mastered the mathematical tools which were required to solve the old problems. The fact that these tools had been available for a long time, may possibly have a message for future generations of economists.

1.4. When satisfactory models of economic equilibrium under uncertainty were presented, this was done independently by Allais [1] and Arrow [3]. That this should happen is almost normal, since the problem had been in the air for some time. It is however remarkable that both models were presented simultaneously, at a colloquium arranged by CNRS in Paris, May 1952.

The two authors took very different approaches to the problem. Allais is closest to the then conventional approach. He assumes that among the goods in the market there are some conditional claims of the kind studied by Böhm-Bawerk. These goods can only be described by probability distributions, and their equilibrium prices must be determined by their stochastic properties. He then gives a complete analysis of the special case in which all contingent claims are normally distributed. In a later version of the paper [2] he remarks that the reader should have no difficulties in generalizing the model for himself. This may be true, but Allais certainly makes the task more difficult than necessary by explicitly rejecting the Bernoulli principle.

Arrow on the other hand accepts the principle, but does not really need it. His starting point is the, by now well known model based on "states of the world". In this model a specific commodity available in two different states, is considered as two different commodities. This simple and ingenious idea makes it possible to reduce the case of uncertainty to the original model of Walras, but with a larger number of commodities. The consistency conditions behind the expected utility theorem are not necessary for this reduction, but they simplify the results.

\section{Arrow's state model}

2.1. Arrow's model does of course represents an important generalization, but it can also be derived as a special case of the classical exchange model.

With the usual notation the Walras model can be described as follows :

$x_{r}^{j}=$ the amount of good $j$ held initially by agent $r$;

$y_{r}^{j}=$ the amount of good $j$ held by agent $r$ in equilibrium;

$p_{j}=$ the equilibrium price of good $j$; 
$u_{r}=$ the utility function which represents the preferences of agent $r$, and has the properties $u^{\prime}(\cdot)>0$, and $u^{\prime \prime}(\cdot)<0$.

The problem of agent $r$ is then

$$
\max u_{r}\left(y_{r}^{1}, y_{r}^{2}, \ldots, y_{r}^{j}, \ldots\right)
$$

subject to the budget condition

$$
\sum_{j} p_{j} y_{r}^{j}=\sum_{j} p_{j} x_{r}^{j}
$$

The familiar solution is given by (1) and the conditions

$$
\frac{\partial u_{r}}{\partial y_{r}^{j}}=\lambda_{r} p_{j} \quad \text { for all } r \text { and } j .
$$

The market clearing conditions are

$$
\sum_{r} y_{r}^{j}=\sum_{r} x_{r}^{j}=x^{j} \quad \text { for all } j
$$

These three sets of equations will under certain conditions determine the general equilibrium, i.e. the equilibrium prices $p_{j}$, and the final holdings $y_{r}^{j}$.

2.2. Let us now interpret $x_{r}^{j}$ as the monetary payoff which agent $r$ will receive from his initial portfolio, if state $j$ occurs, and give a corresponding interpretation to $y_{r}^{j}$. This reduction to a one-good model is convenient, but by no means essential. the form

From the expected utility theorem it follows that the utility function of agent $r$ is of

$$
\sum_{j} f_{j} U_{r}\left(y_{r}^{j}\right)
$$

where $f_{j}$ is the probability that state $j$ shall occur, and $\Sigma_{j} f_{j}=1$. With the assumption of " homogeneous beliefs", i.e. that all agents agree on the probabilities assigned to the different states, the three sets of conditions can be written as follows:

$$
\begin{aligned}
& \sum_{j} p_{j} y_{r}^{j}=\sum_{j} p_{j} x_{r}^{j} \\
& f_{j} u_{r}^{\prime}\left(y_{r}^{j}\right)=\lambda_{r} p_{j} \\
& \sum_{r} y_{r}^{j}=\sum_{r} x_{r}^{j}=x^{j}
\end{aligned}
$$

2.3. From ( $\left.3^{\prime}\right)$ it follows that the superscript $j$ really is redundant. A state is completely described by the value assumed by the stochastic variable $x^{j}$, i.e. the total payoff if state $j$ occurs. Solving the equations $\left(2^{\prime}\right)$, subject to $\left(3^{\prime}\right)$, we obtain a solution which depends only on $x^{j}$, so that we can write $x^{i}=x$ and

$$
p_{j}=p(x), \quad f_{j}=f(x) \text { and } y_{r}^{j}=y_{r}(x) \text {. }
$$

The solution of $\left(2^{\prime}\right)$ and ( $\left.3^{\prime}\right)$ will give $y_{r}(x)$ as a function of $x$, and of the Lagrange multipliers $\lambda_{1}, \ldots, \lambda_{r}, \ldots$. It is easy to see that this solution will give the set of Pareto optimal portfolios. The equations (1) will then determine the Lagrange multipliers, and hence the point in the Pareto optimal set, which corresponds to the competitive equilibrium.

From (2') it follows that $\lambda_{r}^{-1} u_{r}^{\prime}\left(y_{r}(x)\right)$ depends only on the state, hence the expression must, in equilibrium be the same for all agents, so that we can write

$$
\lambda_{r}^{-1} u_{r}^{\prime}\left(y_{r}(x)\right)=U^{\prime}(x) .
$$


Substitution then gives the three sets of conditions in the following form :

$$
\begin{aligned}
& E\left\{p(x) y_{r}(x)\right\}=E\left\{p(x) x_{r}\right\} \\
& p(x)=U^{\prime}(x) f(x) \\
& \sum_{r} y_{r}(x)=x
\end{aligned}
$$

Substitution of (2") into (1") gives

$$
E\left\{U^{\prime}(x) y_{r}(x)\right\}=E\left\{U^{\prime}(x) x_{r}\right\}
$$

These equations together with ( $\left.3^{\prime \prime}\right)$, give a set of equations, which under reasonable assumptions will determine the competitive equilibrium.

2.4. The equations $\left(1^{\prime}\right)$ interpreted literally do of course imply that there must be one price for each state, i.e. the amount one has to pay for a claim to one monetary unit, payable if and only if a particular state occurs. This has led some authors to assume that the model implies that there must exist a competitive market for each state. In many applications the number of states is infinite, often a continuum, so the assumption seems unrealistic. This has led to the suggestion that it is necessary to develop a theory of "incomplete markets", a term often associated with Hirshleifer [9], although the point had been made earlier by others, i.a. by Baudier [4].

From the preceding derivation of Arrow's model it should be clear that the state concept is essentially a technical device to make the mathematical proof rigorous - for a finite number of states. There is no need to trade claims contingent on the occurrence of any single state. This is brought out by (4), which shows that only expectations over states are relevant.

\subsection{The situation can be summed up as follows :}

In the initial situation agent $r$ holds a portfolio described by a stochastic variable $x_{r}$. The expected utility he assigns to this portfolio is $E\left\{u_{r}\left(x_{r}\right)\right\}$, and depends only on $x_{r}$

The market value of the initial portfolio of agent $r$ will depend on the stochastic relationship between $x_{1}, \ldots, x_{r}, \ldots$, not on the stochastic properties of $x_{r}$ alone.

In the final situation agent $r$ will hold a portfolio described by the stochastic variable $y_{r}(x)$, determined by $\left(2^{\prime \prime}\right)$ and $\left(3^{\prime \prime}\right)$. He will assign a higher utility to this portfolio than to his initial holding - except in trivial cases.

From $\left(1^{\prime \prime}\right)$ it follows that the market values of the final and initial portfolio are equal. It also follows that the market value depends only on the stochastic variable $x$.

Hence there should be a need for only one market - a market for uncertain prospects, or portfolios described by stochastic variables. The essential assumption is that an agent can sell his initial portfolio in this market, and buy a Pareto optimal portfolio. The form of Pareto optimal portfolios is given by (2") and ( $\left.3^{\prime \prime}\right)$, and an agent's purchasing power is equal to the market value of his initial portfolio. 


\section{The traditional approach}

3.1. Arrow's model has had an extraordinary impact, and has virtually pushed all earlier work into oblivion. In fact Allais seems to have been the last who has tried to reach general results by the older methods. It will be shown that this, once conventional approach can lead to the same results as Arrow's model.

As a starting point we note that the left-hand side of (4) can be interpreted as the marginal utility of a portfolio described by the stochastic variable $y_{r}(x)$. In equilibrium this marginal utility must be proportional to the market value $P\left\{y_{r}\right\}$, of the portfolio. Hence we write

$$
P\left\{y_{r}\right\}=E\left\{U^{\prime}(x) y_{r}(x)\right\}
$$

Summing (4) over all $r$, we obtain on the right-hand side (from (3")) $E\left\{x U^{\prime}(x)\right\}$, which can be interpreted as the total value of all portfolios in the market. It is then natural to write :

$$
P\{x\}=E\left\{x U^{\prime}(x)\right\}=\int_{0}^{\infty} x U^{\prime}(x) f(x) d x
$$

for this value. It is worth noting that marginal utility and probability density enter (5) in a symmetrical manner.

3.2. It seems reasonable to assume that the total value is independent of how initial endowments are distributed among the agents. This implies that the following condition

$$
P\{x\}=\Sigma_{r} P\left\{x_{r}\right\}
$$

must hold for any partition of the stochastic variable $x$. Clearly (6) means that there are no arbitrage possibilities in the market, i.e. one cannot make a risk-free profit by buying a portfolio, splitting it up, and selling the parts separately.

The problem is now to find a mapping from a set of stochastic variables to the real line, which has the additivity property expressed by (6). To see that a mapping of this kind exists, it is sufficient to observe that

if $\Sigma_{r} x_{r}=x$

$$
\sum_{r} E\left\{x_{r} e^{i x}\right\}=E\left\{x e^{i t x}\right\}=\int_{0}^{\infty} e^{i t x} x f(x) d x
$$

3.3. To obtain a convenient notation we shall write

$$
\begin{aligned}
& \varphi\left(t_{1}, \ldots, t_{r}, \ldots\right)=E\left\{\exp i \sum_{r} t_{r} x_{r}\right\}, \\
& {\left[\frac{\partial \varphi}{\partial t_{r}}\right]_{t_{1}=\ldots t_{r} .}=t=\varphi_{r}^{\prime}(t)}
\end{aligned}
$$

and

$$
\varphi(t, t, \ldots)=\varphi(t)
$$

Setting

$$
P\{x\}=\varphi^{\prime}(t) \text { and } P\left\{x_{r}\right\}=\varphi_{r}^{\prime}(t)
$$

it is easy to verify that (6) is satisfied. 
With this notation $\varphi$ is the Fourier transform of the density, which always exists, and can be interpreted as a moment generating function. If moments exist, this makes it possible to express the value assigned to a stochastic variable in terms of moments, an idea which was very popular at the pre-Arrow period in the economics of uncertainty.

3.4. The assignement of values which we have found is derived from the fundamental property of the Fourier transform. Addition of independent stochastic variables corresponds to multiplication of their Fourier transforms. As we have seen, this result, with minor modifications, carries over to the case of stochastic dependence.

Clearly any linear functional of the functions defined by (7) will satisfy (6). From the Riesz representation theorem it follows that for any continuous linear functional $T(g)$ there exists a function $V(t)$ such that

Hence

$$
T(g)=\int_{-\infty}^{+\infty} g(t) d V(t)
$$

$$
P\{x\}=K \int_{-\infty}^{+\infty} \varphi^{\prime}(t) V^{\prime}(t) d t
$$

where $K$ is some constant, gives the most general continuous mapping which satisfies (6). In (8) we have written $V^{\prime}(t) d t$ for $d V(t)$. If no derivative exists, we shall interpret $V^{\prime}(t)$ as a "generalized function".

3.5. Substituting the expression for $P\{x\}$ given by (8) into (5), we obtain

$$
K \int_{-\infty}^{+\infty} \varphi^{\prime}(t) V^{\prime}(t) d t=\int_{0}^{\infty} x U^{\prime}(x) f(x) d x
$$

From the definition of the Fourier transform it follows that

$$
\varphi^{\prime}(t)=i \int_{0}^{\infty} x f(x) e^{i t x} d x
$$

Taking the inverse transform, we find

$$
x f(x)=\frac{1}{2 \pi i} \int_{-\infty}^{+\infty} \varphi^{\prime}(t) e^{-i t x} d t
$$

Substituting this expression for $x f(x)$ in the right-hand side of (9), and absorbing the constant factors into $K$, we obtain

$$
K \int_{-\infty}^{+\infty} \varphi^{\prime}(t) V^{\prime}(t) d t=\int_{0}^{\infty} U^{\prime}(x) \int_{-\infty}^{+\infty} \varphi^{\prime}(t) e^{-i t x} d t d x
$$

If the order of integration can be reversed, i.e. under the conditions of Fubinis theorem, the equation can be written

$$
\int_{-\infty}^{+\infty} \varphi^{\prime}(t)\left\{K V^{\prime}(t)-\int_{0}^{\infty} U^{\prime}(x) e^{-i t x} d x\right\} d t=0
$$

A solution of this equation is

$$
V^{\prime}(t)=\frac{1}{K} \int_{0}^{\infty} U^{\prime}(x) e^{-i t x} d x
$$

For the sake of symmetry we shall write $V^{\prime}(t)=\omega^{\prime}(-t)$, so that $\omega^{\prime}(t)$ is the Fourier transform of $U^{\prime}(x)$. Substitution into (9) then gives

$$
\int_{-\infty}^{+\infty} \varphi^{\prime}(t) \omega^{\prime}(-t) d t=\int_{0}^{\infty} x f(x) U^{\prime}(x) d x
$$


3.6. This formula is just the classical Parseval's theorem (see i.a. [13]), and could have been written down immediately, and (10) could have been found by using the uniqueness property. The right-hand side of (11) was derived simply and directly from Arrow's state model, which rests on assumptions which many economists have found difficult to accept. The left-hand side was derived after more complicated mathematical manipulations, but rests on the simple assumptions that any asset which can be described by a stochastic variable, will have a market value and that in equilibrium these values will be additive. Earlier generations of economists seem to have accepted these assumptions, almost without questioning. It is interesting - and comforting - that the two sets of assumptions lead to the same result.

From (4) and (7) it follows that the market value of the portfolio held initially by agent $r$ is

$$
P\left\{x_{r}\right\}=\int_{-\infty}^{+\infty} \varphi_{r}^{\prime}(t) \omega^{\prime}(-t) d t=\int_{0}^{\infty} x_{r} U^{\prime}(x) f\left(x, x_{r}\right) d x d x_{r}
$$

On the right-hand side $f\left(x, x_{r}\right)$ is the joint density of the stochastic variables representing the whole market, and the initial portfolio of agent $r$.

In $(11)$ and $(12) \omega^{\prime}(t)$ is the Fourier transform of the marginal utility $U^{\prime}(x)$. In the more classical theory of Fourier analysis the transform does not always exist, but in the more general theory created by Schwartz [14], it will exist as a "generalized function".

3.7. Formula (12) may give some insight into the formation of prices in a market. It may also be useful for computational purposes.

As an illustration we shall consider the special case in which the preferences of all agents can be represented by quadratic utility functions, i.e. when

$$
u_{r}^{\prime}(x)=1-\frac{x}{a_{r}} \quad \text { for all } r
$$

It is easy to show that in this case we have

$$
U^{\prime}(\mathrm{x})=A-x, \quad \text { with } A=\sum_{r} a_{r}
$$

Substitution in the right-hand term of $(12)$ then gives

$$
P\left\{x_{r}\right\}=E\left\{A x_{r}-x x_{r}\right\}=(A-E\{x\}) E\left\{x_{r}\right\}-\operatorname{cov} x x_{r}
$$

which is a familiar result from portfolio theory.

Considering the middle term in (12), we observe that, in the ordinary sense the Fourier transform of $U^{\prime}(x)=A-x$ does not exist, but it exists as a generalized function, and we have

$$
\omega(t)=A \delta(t)+\delta^{\prime}(t)
$$

Here $\delta(t)$ is the Dirac function, which has the properties

$$
\begin{aligned}
& \int_{-\infty}^{+\infty} g(x) \delta(x) d x=g(0) \quad \text { and } \quad \int_{-\infty}^{+\infty} g(x) \delta^{\prime}(x) d x=-g^{\prime}(0) \\
& \text { As } \varphi^{\prime}(0)=E\left\{x_{r}\right\} \quad \text { and } \varphi^{\prime \prime}(0)=E\left\{x x_{r}\right\}=\operatorname{cov} x x_{r}
\end{aligned}
$$

it follows immediately that the middle term is equal to the right-hand side. 


\section{Incomplete markets}

4.1. It is not easy to trace the origin and the increasing popularity of the concept " incomplete market". In the discussion following the paper by Baudier ([7] pp. 305-310) it seems to have been agreed that Arrow's state model, in spite of its elegance, was too complex to be realistic. In the real world one would expect a group of agents to organize their exchanges of assets or liabilities in a simpler way - through the rules they lay down, and the institutions they set up. Such arrangements will in general lead to an equilibrium, which however may not necessarily be Pareto optimal.

Baudier argued that such sub-optimal arrangements are of considerable practical interest, and deserve further study. Others maintained that arrangements of this kind will in some sense be trancient, since a group of rational agents must be expected, sooner or later, to find their way to a Pareto optimum - through bargaining or institutional changes.

4.2. A simple arrangement would be to set up markets where the agents could buy and sell fractions of the initial portfolios. If there are $n$ agents in the market, it will be necessary to create $n$ markets. A smaller number is required if the initial portfolios are made up of $m<n$ identifiable assets, such as stock in $m$ different companies.

In general, markets of this kind will have an equilibrium, but this may not be a Pareto optimum. An exchange of fractions of portfolios - or of common stock - can lead to a Pareto optimum if the utility functions satisfy some rather restrictive conditions. If these conditions are not satisfied, the market is said to be "incomplete", because the equilibrium can be brought closer to a Pareto optimum, if markets are set up for new securities, such as preferred stock, bonds and options. It seems reasonable to assume that only transactions costs can prevent a Pareto optimum to be reached through the creation of additional markets. The question is however not simple, and we shall not discuss it in any detail in the present context.

4.3. The traditional approach, discussed in Section 3, assumes from the start that the market is complete. The approach has its origin, at least in part, in insurance. In this industry it seems quite natural to assume that the market can quote a premium for cover of any risk, described by a well defined probability distribution with finite expectation. It is also generally assumed that the risk can be split up, or merged with others, and reinsured in the market at the going rate.

The exceptions, the uninsurable risks, seem to occur only when some element of "moral hazard" is present. In these cases the probability distribution describing the risk depends on the insurance cover, and is not well defined.

4.4. Similar situations evidently exist, also outside the insurance industry. If both an entrepreneur and his workers are risk averse, a conventional wage contract will be sub-optimal, since it implies that all risk is carried by the entrepreneur. Clearly both parties could be made better off with some profit-sharing arrangement, but it is possible that the workers would not accept this unless they get some say in the management.

In this example the labor market may be said to be incomplete. The example does however indicate that the concept "incomplete market" is more closely connected with 
incentives and moral hazard than with the large number of states in Arrow's model. The owner of a well insured factory may not have the incentive to make desperate efforts to put out a fire. Things might be different if he had to fight for his economic survival.

It may be appropriate to close by noting that the possibilities in existing markets can be overlooked. No boutique will sell a woman a black dress with accessories, to be delivered only if she should become a widow within a year. Should however a woman want a conditional claim of this kind in her portfolio, any insurance salesman will be glad to arrange it for her.

\section{REFERENCES}

1. ALLAIS, M.: "Généralisation des théories de l'équilibre économique général et du rendement social au cas du risque ", Colloques Internationaux du CNRS, XL, pp. 81-120, Paris, 1953.

2. ALLAIS, M. : “L'extension des théories de l'équilibre économique général et du rendement social au cas du risque ", Econometrica, 1953, 269-290.

3. ARROW, K. J. : "Le rôle des valeurs boursières pour la répartition la meilleure des risques", Colloques Internationaux du CNRS, XL, pp. 41-88, Paris, 1953.

4. BAUDIER, E. : "Minimax behaviour and price prediction", in [7], 283-305.

5. BERNOULLI, D. : "Specimen theoriae novae de mensura sortis", St. Petersburg, 1738. English translation Econometrica, 1954, 23-36.

6. BÖHM-BAWERK, E.: Rechte und Verhältnisse vom Standpunkte der Volkswirtschaftlichen Güterlehre, Innsbruck, 1881.

7. BORCH, K., and MOSSIN, J.: Risk and Uncertainty, Macmillan, London, 1968.

8. CZUBER, E. : Wahrscheinlichkeitsrechnung, Teubner, Leipzig, 1902.

9. HIRSHLEIFER, J. : Investment, Interest and Capital, Prentice-Hall, Englewood Cliffs (N.J.), 1970.

10. MARSHALL, A. : Principles of Economics, Macmillan, London, 1890.

11. NEUMANN, J. von, and MORGENSTERN, O.: Theory of Games and Economic Behavior, 2nd edition, Princeton University Press, 1947.

12. RAMSEY, F. P. : "Truth and probability", The Foundation of Mathematics and other Logical Essays, Kegan Paul, London, 1931.

13. RUDIN, W. : Real and Complex Analysis, MacGraw-Hill, New York, 1970.

14. SCHWARTZ, L. : Théorie des distributions, Hermann, Paris, 1957.

15. WALRAS, L. : Eléments de l'Economie Politique pure, Lausanne, 1875-1877. 